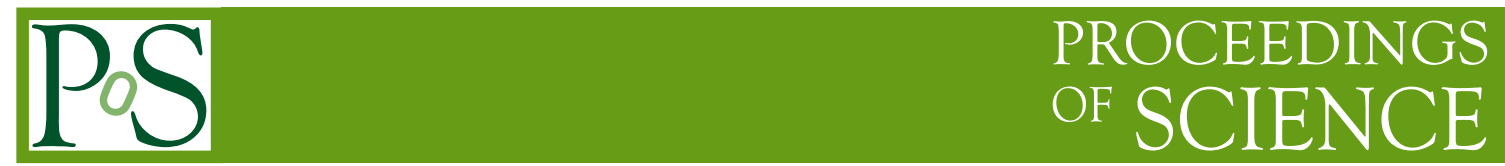

\title{
Galactic and Extragalactic Novae - A Multiwavelength Review
}

\section{Rosa Poggiani* ${ }^{*}$}

Università di Pisa and Istituto Nazionale di Fisica Nucleare, Sezione di Pisa

E-mail: rosa.poggiani@unipi.it

This paper is an updated version of the review [130] about multifrequency observations of novae. I review the observations of Galactic and extragalactic novae over the electromagnetic spectrum, with an accent on their high energy emission and the role played by new present ground and space based facilities.

The Golden Age of Cataclysmic Variables and Related Objects V (GOLDEN2019)

2-7 September 2019

Palermo, Italy

\footnotetext{
*Speaker.

${ }^{\dagger}$ Corresponding author
} 


\section{Introduction}

Classical novae are cataclysmic variable systems where a white dwarf is accreting material from a secondary star [116], [17], [10]. The nova outburst is produced by a ThermoNuclear Runaway (TNR) on the surface of the white dwarf [17], [10], [172], [51]. Novae are multiwavelenght sources, since their emission extends over the whole electromagnetic spectrum [59], even if they are usually discovered by their optical brightness rise. In the present paper, I review multi-frequency observations of Galactic and extragalactic novae. The availability of a rich pattern of instrumentation and the distances provided by Gaia have opened new investigation windows and revisited some accepted paradigms. Novae have been found to be the origin of a relevant fraction of Galactic lithium. Novae are confirmed sources of high energy $\mathrm{GeV}$ gamma rays and could be also $\mathrm{TeV}$ sources. The relation of novae with cataclysmic variables has been highlighted by the observation of dwarf nova outbursts preceding or following the nova outburst. The Maximum Magnitude Rate of Decline (MMRD) relation has been revisited, thanks to observations of extragalactic novae. At the same time, the Gaia DR2 release has allowed to investigate the distance of galactic novae. The properties of Galactic and extragalactic novae has been reviewed by [42], [157].

\section{Optical Observations}

After the outburst, novae undergo a decline that can be smooth, but can also show dips, oscillations, flares etc. The work by [174] has classified the decline curves into seven prototype classes (Fig. 1): the F-class novae with flat top (2\% of all novae); the C-class with a cusp (1\%); the J-class with jitters (16\%); the P-class with a plateau (21\%); the O-class with oscillations (4\%); the S-class with a featureless decline (38\%); the D-class with dips (18\%). The decline of S-class novae is described by an universal law in the model by [62], [78].

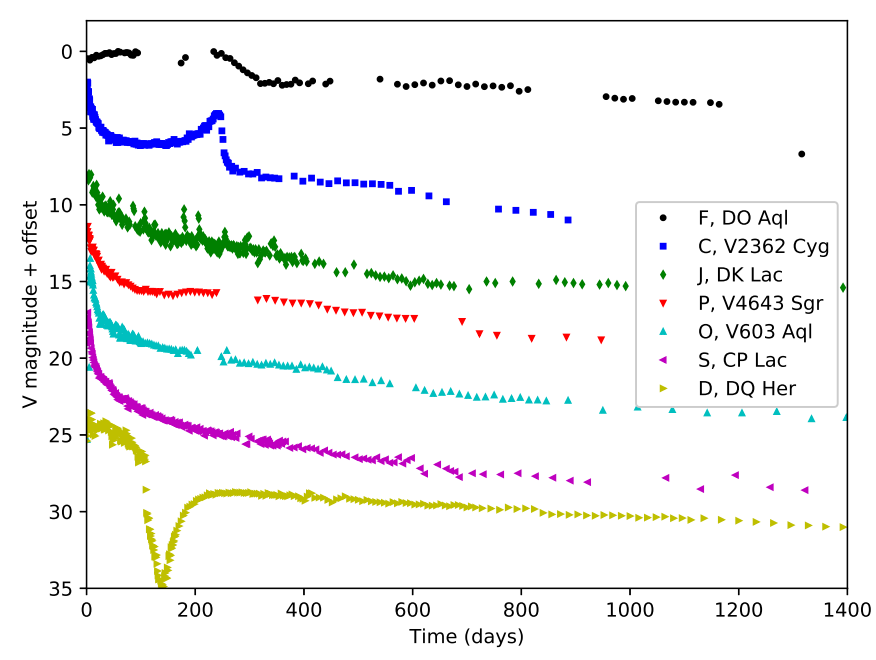

Figure 1: Classification of the light curves of novae, after [174]; data from [174]

A large number of all-sky and high time cadence surveys presently allow to investigate novae whose maximum has been missed, the poorly explored pre-maximum stage and processes occurring at short timescale. Survey observations include both Galactic and extragalactic novae. 
The ground based Optical Gravitational Lensing Experiment (OGLE) has monitored 39 Galactic novae [97]; among them, one third has been discovered during the survey. In addition, the OGLE survey includes 80 post-nova candidates and has measured the orbital periods of 18 systems. The Solar Mass Ejection Imager (SMEI) has monitored 13 galactic novae [69], [70], including systems whose maximum had been missed. Some systems have been observed during the poorly observed pre-maximum stage (see [129] and references therein for a review of the pre-maximum stage). The high temporal cadence survey of M31, M81, M82, NGC 891, NGC 2403 [77] has discovered a new class of faint and fast novae, discussed below, whose properties are not consistent with the MMRD relations.

The original spectral classification by [189], [190], [191] with two classes, Fe II novae and $\mathrm{He} / \mathrm{N}$ novae, according to the presence of Fe II multiplets or He/N lines, has been recently revisited. Fe II novae are dominated by wind ejection and account for the majority, about $80 \%$, of Galactic novae, while He/N novae are dominated by shell ejection. Some novae, the hybrid novae, show spectra that switch from the Fe II to the He/N class. Sample post-outburst spectra of Fe II, He/N and hybrid novae are reported in Fig. 2. He/N novae are brighter and faster than Fe II novae [46] and show an higher concentration around the Galactic plane [37], [46].
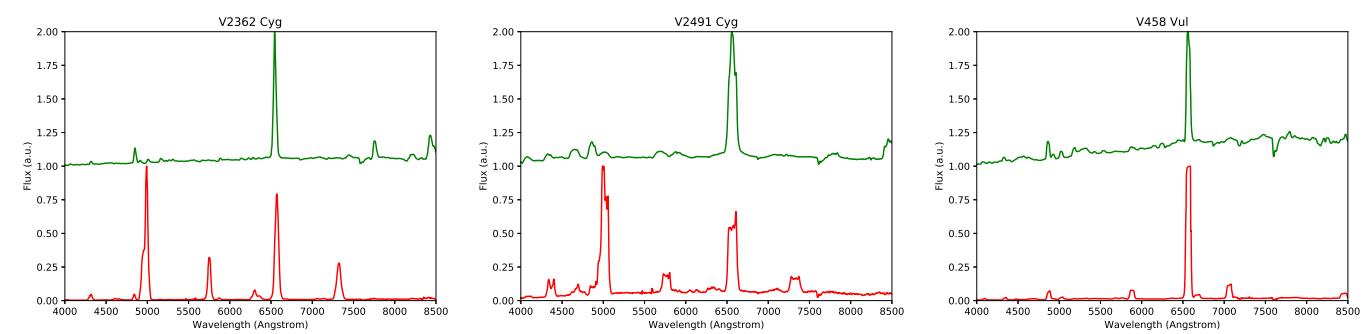

Figure 2: Spectra of the Fe II nova V2362 Cyg [120], He/N nova V2491 Cyg (Poggiani, in preparation). hybrid nova V458 Vul [119] during the initial decline (top curves) and the late stages (bottom curves)

Some recent novae have shown a spectroscopic evolution departing from the above picture. Novae V5558 Sgr [177] and T Pyx (see e.g. [73], [52]) have showed initial spectra consistent with the He/N class, followed by Fe II class spectra. The standard classes of nova spectra can be explained by their production in the ejecta of the white dwarf or in a circumbinary gas envelope surronding the secondary star [194]. The observation of a Fe II or a He/N spectrum is determined by the relative contribution of the above mechanims [194].

Optical spectroscopic investigations of novae have shed light on the contribution of novae to Galactic nucleosynthesis. The presence of lithium has been searched for a long time. Several production mechanisms have been proposed, involving interaction of cosmic rays with interstellar material, Big Bang nucleosynthesis, novae and supernovae. High resolution spectroscopic observations of nova V1369 Cen showed an absorption feature at $6695.6 \AA$, a blue shifted ${ }^{7} \mathrm{Li}$ I $6708 \AA$ transition. at different epochs [75]. Also V5668 Sgr, showed an absorption line corresponding to the blue shifted Li I $6708 \AA$ transition [186]. The blue shifted resonance line of the singly ionized isotope of ${ }^{7} \mathrm{Be}$, that decays to form ${ }^{7} \mathrm{Li}$ with an half life of about fifty days, has been observed in the NUV spectra of nova V339 Del [176]. It has been estimated that novae can explain the amount of ${ }^{7} \mathrm{Li}$ observed in the Galaxy [95]. 
So far, there are a few compilations of nova spectra observed with high time cadence. After the historical Tololo nova atlas [189], [191], the SMARTS Consortium [187], [188] has built a living spectral atlas of novae observable from Southern hemisphere using the ANDICAM dual channel imager and the RC spectrograph at the SMARTS $1.5 \mathrm{~m}$ telescope. The atlas comprises about one hundred novae and has been used to perform systematic studies of nova properties, among them the relation of He II 4686 in He/N novae and the X-ray super-soft stage and the link between the $\mathrm{K}_{s}$ band flux and dust formation [188].

I have been monitoring Northern novae since 2005 at the Cassini $1.5 \mathrm{~m}$ telescope, with the BFOSC Imager/Spectrograph [126], securing optical spectra at the highest temporal cadence allowed by telescope time and weather. The observed sample comprises more than twenty Galactic novae and some extragalactic novae: V1663 Aql [117], V1722 Aql, V809 Cep, V962 Cep, V2362 Cyg [120], V2467 Cyg [121], V2468 Cyg, V2491 Cyg, V2659 Cyg, V407 Cyg, V339 Del, KT Eri, V959 Mon, V2615 Oph, V2670 Oph [122], V2944 Oph, V496 Sct, V612 Sct (ASASSN-17hx) [129], V556 Ser, V5558 Sgr [118], [123], V5584 Sgr [125], [128], V458 Vul [119], V459 Vul [124], the extragalactic novae M31 2009-10b, M31 2010-07a, M31 2011-07 and M33 2010-07a [127]. Some observed novae have shown a peculiar photometric and spectroscopic behavior: V458 Vul is an hybrid nova [119]; V2467 Cyg showed oscillations during the initial decline and an early appearance of nebular lines [121]; V5558 Sgr is a very slow nova with a white dwarf mass at limit to trigger the outburst [118], [123]; V2362 Cyg showed a cusp due a secondary mass ejection [120]; V612 Sct (ASASSN-17hx) is a slow nova with a long pre-maximum stage [129]; M31 2009-10b is one of the brightest novae ever observed in M31 [127]; M33 2010-07a is the first nova in M33 showing a secondary mass ejection [127].

\section{Infrared Observations}

In addition to abundances, infrared observation of novae provide information about dust production and the geometry of ejecta [20]. The spectra of FH Ser by [58] showed an infrared brightening in correspondence to the optical fading, suggesting that dust formation had occurred. The observations in the near infrared region corresponding to $\mathrm{J}, \mathrm{H}, \mathrm{K}$ bands are used to classify novae [7], as in the optical classification scheme above. The Fe II and He/N classes remain distinct classes also in the near infrared. Carbon lines are present only in Fe II novae, but not in $\mathrm{He} / \mathrm{N}$ novae. On the other hand, hydrogen Paschen and Brackett lines appear in both of them. Near infrared $\mathrm{CO}$ emission can be used to estimate the ${ }^{13} \mathrm{C}$ yield and the effect on the chemical evolution of Galaxy [7]. The $\mathrm{CO}$ emission is investigated through the first overtones, starting from the $\mathrm{K}$ band [7]. The infrared region above $3 \mu \mathrm{m}$ is relevant for dust investigations [53], whose composition spans silicates, amorphous carbon, $\mathrm{SiC}$, hydrocarbons [53]. Dust formation is often, but not necessarily, accompanied by a dip in the optical light curve, and by an infrared brightening caused by the heating and the re-emission of dust particles. Nova V705 Cas is an example. The optical and infrared light curves are shown in the left and right panels of Fig. 3. The dip in the optical curve is produced by the formation of an optically thick carbon dust shell [90], that corresponds to a maximum in the infrared light curve.

In addition to showing that novae are dust grain producers, infrared observations have shown the bipolar nature of the ejection of nova shells [18], [19], [141]. 

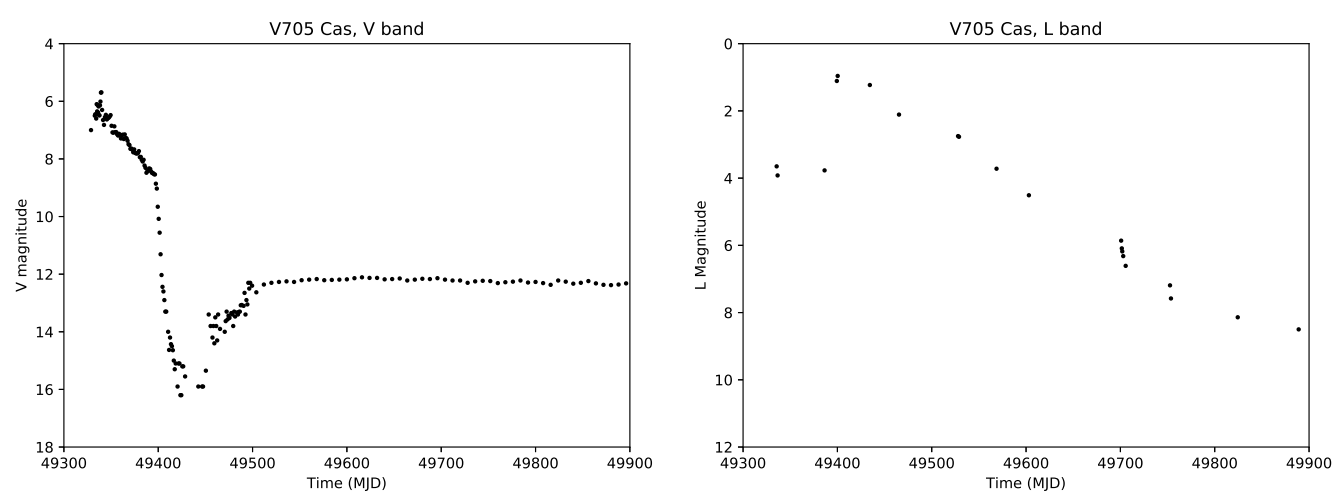

Figure 3: The V band light curve (left, data from [174]) and the L band light curve (right, data from [90])

\section{Radio Observations}

The radio emission of novae evolves over time scales of years, much longer than the optical bright phase [138]. The multifrequency radio curves of V1500 Cyg [68] are shown in Fig. 4.

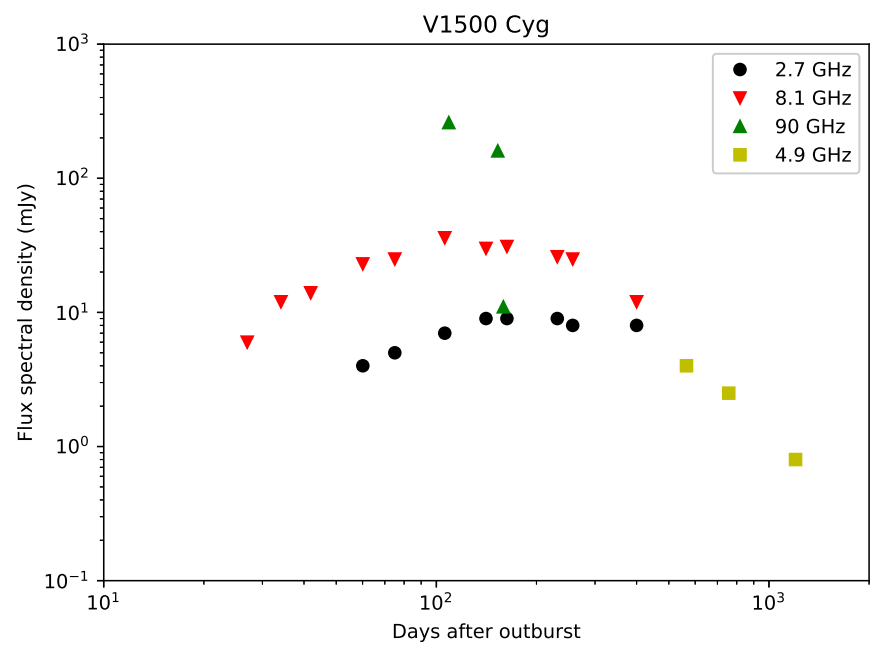

Figure 4: The multifrequency radio light curves of V1500 (data from [68])

The main mechanism is the thermal bremsstrahlung of the ejecta, that can be modelled by a spherical and isothermal shell of ionized gas with a power law density gradient [10] and is used to estimate the mass of the ejecta [138].

High resolution radio observations have resolved the ejecta of some novae, that are non spherical and can exhibit a bipolar geometry, see for example V1974 Cyg [54], the 2006 outburst of RS Oph [104], [9], the recent gamma ray nova emitting nova V959 Mon [24]. The E-nova Project at the Very Large Array (VLA) monitors novae in the region between a few $\mathrm{GHz}$ and some hundreds $\mathrm{GHz}$, starting observations within a few days after the eruption. V407 Cyg, the first nova detected in gamma rays, is among the novae in the program [23]. 


\section{X-ray Observations}

$\mathrm{X}$-ray emission in novae can be produced via thermal emission from the white dwarf, when ejecta are becoming optically thin in the SuperSoft X-ray Source phase (SSS), via the shock of ejecta on surroinding material or from accretion during quiescence. The first observations with EXOSAT [105] and ROSAT [80] showed flux variations during the nova outburst and a spectral evolution towards a supersoft phase. The hard and soft components are associated to shocks within the ejecta and to the nuclear burning on the white dwarf surface.

Swift has produced light curves of novae from the very early phase for a large number of novae in the Galaxy and in the close galaxies M31, LMC, SMC [102], [111]. The 0.3-10 keV light curve of RS Oph [145] is reported in Fig. 5.

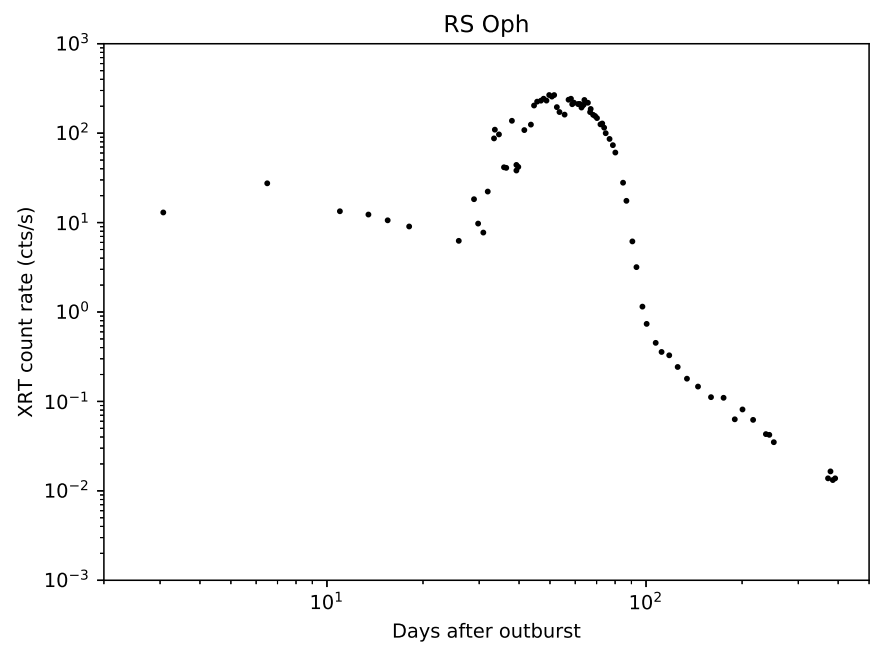

Figure 5: The 0.3-10 keV light curve of RS Oph; data from [145]

The 2006 outburst of RS Oph showed shocks in the ejecta [8], [171], [110], with radio [104] and HST observations [9] pointing to a bipolar geometry with an equatorial ring. After the nova outburst, the keV X-ray flux declined and later peaked at about day 30, when SSS phase began. The photosphere shrinking and optical depth decreasing exposed the hotter layers. The burning stopped on day 80 and caused the X-ray fading. The super-soft phase, often associated with the optical [Fe X] $6374 \AA$ Alne, has been detected in several novae [145]. Fast Galactic and M31 novae show high ejection velocities and early turn-on and turn-off of the SSS phase [65].

After the early observations of the thermal bremsstrahlung continuum, a rich pattern of transitions is being observed with the present high resolution instruments [106]. The high resolution grating X-ray spectra of XMM-Newton and Chandra show the continuum and the white dwarf absorptions or broad emission lines or a combination of the above features [107], [103]. Collisional plasmas can explain the spectra outside the super-soft phase [103]. Shocks produce emission before the onset of the SSS stage, while the later emission is caused by the radiatively cooling thin ejecta [103]. The turn on and off times of the SSS phase have been modeled by [62], [78] [63]. 


\section{Gamma Ray Observations}

Gamma rays from novae were expected in the $\mathrm{MeV}$ region, in association with positron annihilation and nuclear de-excitation of nitrogen, oxygen and sodium [27], [67]. Surprisingly, the first detection of gamma rays from a nova occurred at high energies, when the Fermi-LAT instrument observed the 0.1 to $10 \mathrm{GeV}$ emission of the symbiotic nova V407 Cyg [1]. The gamma ray emission is explained by the interaction of material in the nova shell with the environmental medium of the secondary. Since V407 Cyg is a symbiotic nova, ejecta expand in a circumstellar wind and particles acceleration occurs in a blast wave. Fermi-LAT detected $\mathrm{GeV}$ gamma ray emission in other classical novae: V959 Mon, V1324 Sco and V339 Del [2], V1369 Cen and V5668 Sgr [21], V407 Lup [22], ASASSN-16ma [81], ASASSN-17mt [83], V906 Car/ASASSN-18fv [76], V392 Per [84], V1707 Sco [85], V3890 Sgr [14]. The GeV light curve of V959 Mon is shown in Fig. 6.

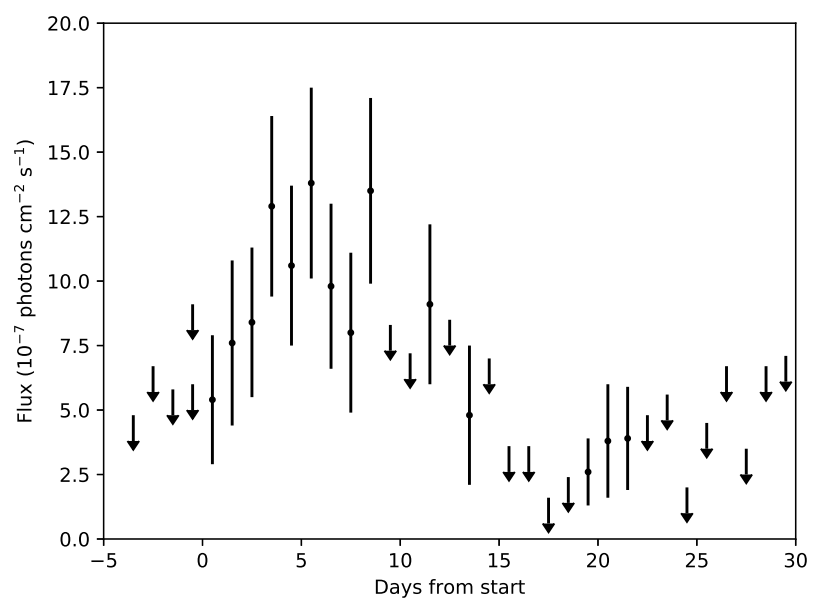

Figure 6: Fermi-LAT light curve (>100 MeV) of V959 Mon [2]; data from [2]

The observation of $\mathrm{GeV}$ gamma ray emission in novae suggests that all of them are potential gamma ray emitters, but their emission could be not detectable due to their distance. The FermiLAT instrument detected gamma rays from six novae, out of the 69 novae discovered during the same period [96]. Novae with a magnitude $\mathrm{R} \leq 12$ and within $8 \mathrm{kpc}$ are potentially detectable by the Fermi-LAT instrument [96].

Gamma ray emission in novae has been discussed by the hadronic and the leptonic models [2]. In the former, nova ejecta interact with nuclei in the environment (novae) or with the stellar wind (symbiotics), producing neutral pions decaying into photons. In the latter, gamma rays are produced in the interaction of accelerated electrons with photons via inverse Compton or with atoms via bremsstrahlung.

Novae emitting gamma rays allow to investigate relativistic particle acceleration at shocks [92]. The high density ejected material produces radiative shocks. The fraction of the shock power spent in the acceleration of relativistic particle is governed by the ratio of gamma ray and optical luminosities. The model predicts X-ray emission in the range from 10 to $100 \mathrm{keV}$, potentially detectable by NuSTAR, in coincidence with GeV emission [92]. Only a small fraction of the 
luminosity lies in the NuSTAR energy band, but with a clear signature, a zero spectral slope [184]. The high energy emission of novae is mostly produced from particles accelerated up to $100 \mathrm{GeV}$ at the reverse shock and linked to hadronic interactions in the cooling layer downstream of the shock [89]. Gamma ray emission does not show a clear correlation to the optical emission, with the exception of a few novae [101]. The correlation of gamma rays and optical emission from ASASSN-16ma pointed to the role of reprocessed emission from shocks [82].

The shocks and the relativistic particle acceleration in a nova outburst can potentially produce gamma ray emission in the $\mathrm{TeV}$ region, up to $100 \mathrm{TeV}$ [93], the region of ground based atmospheric Cherenkov detectors, such as MAGIC or the future Cherenkov Telescope Array CTA [3]. The Cherenkov instrument MAGIC has monitored V339 Del in the energy region above $50 \mathrm{GeV}$, with negative detections [4]. The $\mathrm{TeV}$ gamma rays could also be associated with $\mathrm{TeV}$ neutrinos, potentially detectable with IceCube [169].

\section{Galactic and Extragalactic Nova Populations}

To date, more than four hundreds Galactic novae are known, according to the catalogs by [49], [48], [132], [133], [100]. The Galactic nova rate has been reviewed by [156], who proposed a rate of $50_{-23}^{+31} \mathrm{yr}^{-1}$. A collection of the estimated Galactic nova rates is reported in Table 1.

\begin{tabular}{lr}
\hline Authors & Rate $\left(\mathrm{yr}^{-1}\right)$ \\
\hline Lundmark 1935 [88] & 50 \\
Allen 1954 [5] & 100 \\
Kopylov 1955 [79] & 50 \\
Sharov 1972 [167] & 260 \\
Liller and Mayer 1987 [86] & $73 \pm 24$ \\
Della Valle 1988 [36] & $15 \pm 5$ \\
Ciardullo et al. 1990 [26] & $11-46$ \\
van den Bergh 1991 [183] & 16 \\
Della Valle and Livio 1994 [43] & $15-24$ \\
Hatano et al. 1997 [64] & $41 \pm 20$ \\
Shafter 1997 [147] & $35 \pm 11$ \\
Shafter 2002 [149] & $36 \pm 13$ \\
Matteucci et al. 2003 [91] & 25 \\
Darnley et al. 2006 [33] & $34_{12}^{+15}$ \\
Mroz et al. 2015 [97] & $13.8 \pm 2.6$ \\
Shafter 2017 [156] & $50_{-23}^{+31}$ \\
\hline
\end{tabular}

Table 1: Galactic nova rates

The study of Galactic novae is affected by the interstellar absorption. The effect can be reduced by observing the population of novae in other galaxies. The properties of extragalactic nova populations have been reviewed by [154], [157]. The surveys has produced nova rates for M31, M33, LMC, SMC, M87, that will be discussed below, and other close galaxies.

The nova rate in M31 has been investigated in detail. The spectroscopic survey of the M31 bulge by [180] was consistent with the spectral properties of Galactic novae. The survey by [151] reported the fraction of Fe II and He/N novae, $82 \%$ and $18 \%$ respectively, consistent with Galactic 
novae. The existence of two distinct nova populations in M31, in disk and bulge, was suggested by [33]. The saturation of photographic plates used in the early surveys [71], [6], [135], [136], [137] precluded the detection of novae in M31 nucleus. Novae can appear in any part of M31 [26], with a concentration in the halo and bulge [25], [15]. The estimated nova rates in M31 are summarized in Table 2.

\begin{tabular}{lr}
\hline Authors & Rate $\left(\mathrm{yr}^{-1}\right)$ \\
\hline Hubble 1929 [71] & 30 \\
Arp 1956 [6] & $26 \pm 4$ \\
Capaccioli et al. 1989 [15] & $29 \pm 4$ \\
Shafter and Irby 2001 [158] & $37_{-8}^{+12}$ \\
Darnley et al. 2006 [33] & $65_{-15}^{+16}$ \\
\hline
\end{tabular}

Table 2: M31 nova rates

The estimates of the nova rate in M33 are reported in Table 3. The M33 nova rate should be higher than the M31 one, since it is an early type galaxy [38]. To date, about fifty novae have been observed in M33, but only a small fraction has received a spectroscopic classification. The survey of M33 novae by [152] showed that 5 and 2 novae out of 8 were He/N and Fe II novae, respectively, differently from the Galactic and M31 novae.

\begin{tabular}{lr}
\hline Authors & Rate $\left(\mathrm{yr}^{-1}\right)$ \\
\hline Sharov 1993 [168] & $<0.4$ \\
Della Valle 1994 [38] & $4.7 \pm 1.5$ \\
Williams and Shafter 2004 [193] & $2.5_{-0.7}^{+1.0}$ \\
\hline
\end{tabular}

Table 3: M33 nova rates

The rate of LMC novae is of the order of a few novae per year: 2-3 $\mathrm{yr}^{-1}$ [60], $2 \pm 1 \mathrm{yr}^{-1}$ [16], $2.5 \pm 0.5 \mathrm{yr}^{-1}$ [40], 2.4 $\pm 0.8 \mathrm{yr}^{-1}$ [98]. In particular, the work by [98] has detected 20 novae in LMC, among them 13 new discoveries. The survey of LMC novae by [153] has investigated the nova population, observing that the decline rates are faster than those of Galactic and M31 novae. In addition, the survey found a comparable fraction of novae of $\mathrm{Fe}$ II and $\mathrm{He} / \mathrm{N}$ novae [153]. The fast decline times, the larger fraction of $\mathrm{He} / \mathrm{N}$ and the higher fraction of recurrent novae suggests that LMC novae contain more massive white dwarfs. About SMC, seven novae were discovered in the survey by [98], with an estimated rate of $0.9 \pm 0.4 \mathrm{yr}^{-1}$. The large fraction of He/N novae in M33 and LMC is explained by their young stellar population, leading to more massive white dwarfs and an higher number of recurrent novae.

The estimations of the rate in M87 span a broad range: $91 \pm 34 \mathrm{yr}^{-1}$ [148]; $154_{-19}^{+23} \mathrm{yr}^{-1}$ [31]; $363_{-45}^{+33} \mathrm{yr}^{-1}[162]$

The behavior of the nova rate against the luminosity of the host galaxy has been investigated by different authors [44], [148]. The nova rates in different galaxies are compared through the Luminosity Specific Nova Rates (LSNR), with a normalization to the investigated stellar mass [154] via the galaxy K-band luminosity. The rates in galaxies belonging to different Hubble types has been presented by [196], [91]. The nova rate is proportional to the K-band luminosity of the galaxy (Fig. 7). 


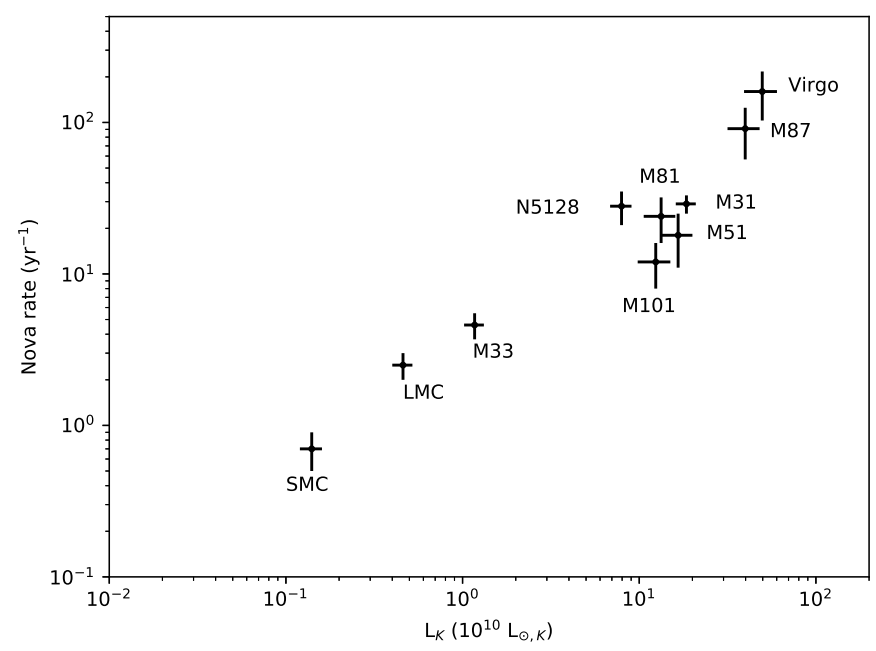

Figure 7: Nova rate in galaxies versus the K-band luminosity; data from [91]

Some historical novae have been superluminous, for example V1500 Cyg [39], nova LMC 1991 [39], [144], SN 2010U in NGC 4214 [32], M31 2007-11d [150], M31 2009-10b [127]. While V1500 Cyg could be an hybrid nova [150], all extragalactic novae belong to Fe II class, that should be associated to less massive white dwarfs. Probably the superuminous novae harbour cool dwarfs accreting material at a very slow rate [32].

\section{Recurrent Novae}

Recurrent novae show outbursts with recurrence times of the order of decades [10]. To date, there are 10 known recurrent Galactic novae [140], 4 in LMC [108], 12 in M31 [155]. Recurrent novae are grouped into three classes. CI Aql, T Pyx, IM Nor have orbital periods shorter than one day and main sequence secondary stars; U Sco, V394 CrA and V2487 Oph have orbital periods of about one day and evolved secondaries; T CrB, V3890 Sgr, RS Oph, V745 Sco have orbital periods of the order of years and red giant secondaries. The majority of recurrent novae are He/N novae. The photometric evolution of known Galactic recurrent novae has been presented by [140]. The light curve of RS Oph [140] is shown in Fig. 8.

The recurrence time is governed by the mass of the white dwarf and by the accretion rate [195]. Recurrent novae generally show an high rate of mass transfer [108] and has been suggested as possible progenitors of type Ia supernovae [175], [155], [173].

During the recent years, recurrent novae T Pyx, V745 Sco and V3890 Sgr underwent outbursts and were monitored at all wavelengths (see e.g. [73], [52], [113], [109]).

The fraction of recurrent novae in M31 is of the order of a few percent [155]. Nova M31 2008-12a has a recurrence time of one year and is a supernova Ia progenitor candidate [66]. Several outbursts have been recorded with ground and space based instruments and are reported by [34], [74], [11]. 


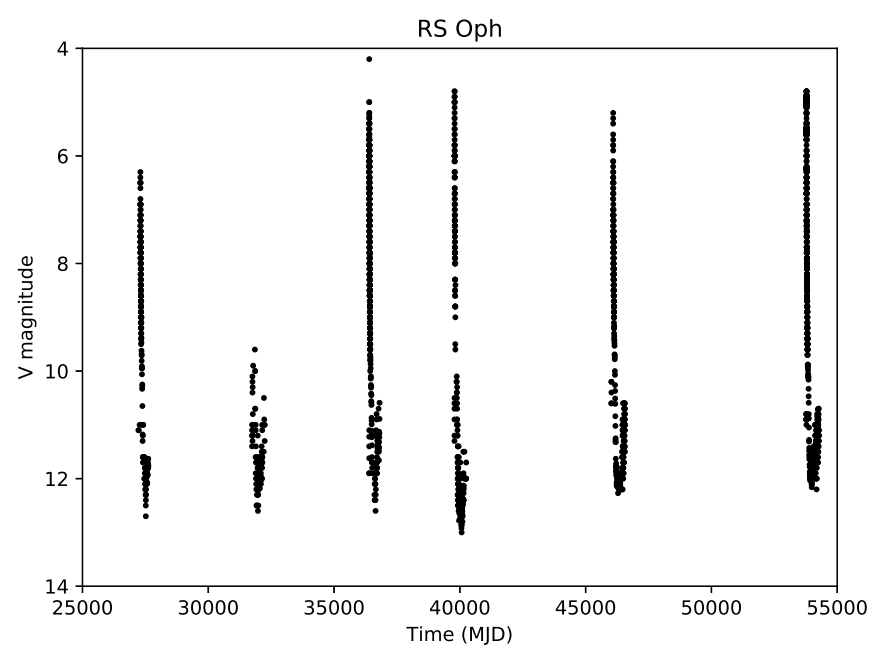

Figure 8: The historical light curve of RS Oph; data from [140]

\section{Novae and Cataclysmic Variables}

The orbital period has been measured only for a small fraction of known novae. The distribution of measured periods, built using the data by [132], [133], is shown in Fig. 9, where novae with evolved secondary stars and periods above 10 hours have been excluded. The distribution of nova periods has a peak between 3 and 4 hours, above the period gap of cataclysmic variables. Novae show an high mass accretion rate onto the white dwarf [179].

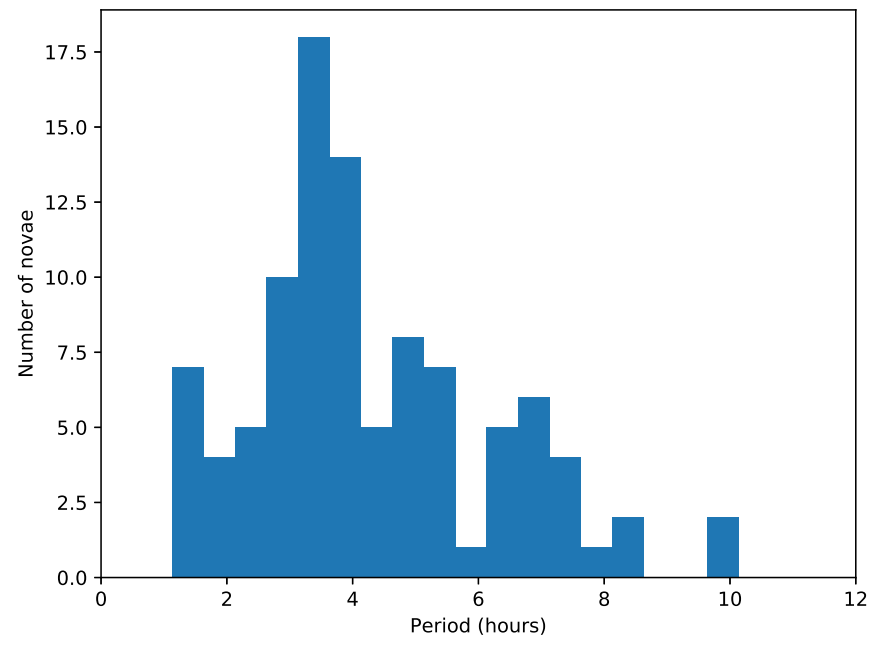

Figure 9: Distribution of the orbital periods of Galactic novae; data from [133]

The evolution of novae over long time scales has been discussed by [115]. The pre-outburst light curves presented by [134] and [30] show that the quiescent magnitude of the majority of novae does not change before and after the eruption [30]. It has been proposed that novae could enter hibernation in a state with low mass transfer rate [159]. The combination of gravitational 
wave emission and magnetic braking during the hibernation stage produce a shrinking of the orbit, against the detachment of the secondary star from its Roche lobe. The white dwarf irradiation mantains a relatively high brightness for decades after the outburst, later fading and varying the accretion rate, with dwarf nova outbursts occurring [159]. Novae are expected to show a cyclical evolution between high and low mass transfer states [159], with hibernation duration above some hundreds thousands years. The hibernation model predictions are in agreement with the observed decline rates of old novae [50] and the observation of shells around the dwarf novae Z Cam [160], AT Cnc [161] (that underwent a nova eruption about three centuries ago [165]), the dwarf nova in Te 11 (associated to the nova 483 CE in Orion) [94], the nova-like systems V1315 Aql [139] and IPHASX J210204.7+471015 [61]. Some novae have shown dwarf nova outbursts: V1213 Cen [99], V1047 Cen [57], Nova Sco 1437 AD [163].

The recover of old novae in catalogs of cataclysmic variables [48] is made difficult by the frequent lack of spectroscopic identification. Observatory plate archives are being mined by [178], [72], [114]. The selection criteria for old novae combine multicolour photometry and spectroscopic observations [178], [114]. Old novae are selected by their position in the color-color diagram, apart from the main sequence curve [178], and later spectroscopically observed to check their classification.

\section{Novae Distances}

The distances of novae have been determined using the nova expansion parallax [47], [170], the Maximum Magnitude Rate of Decline (MMRD) relations [29], [45], [35], [47], [143], the identity of the absolute magnitude achieved 15 days after the maximum [13], [15], [28], [47], [182]. The nova expansion parallax [47], [170] of shells around novae measured years or decades after the outburst must take into account the system inclination and non isotropic mass ejection [47], [185]. While the identity of the absolute magnitude after 15 days is supported by the observation of M87 novae [166], the MMRD relations have been revisited. The high time cadence survey of M31, M81, M82, NGC 891, NGC 2403 by [77] has discovered a new class of fast and faint novae, whose luminosity and decay times are not consistent with the standard MMRD relations. The majority of novae were $\mathrm{Fe}$ II novae, not He/N, and accounted for about 40\% of novae in M87 [164]. The faint and fast novae can be associated to massive white dwarfs [77], [195], [87]. A new distance estimation method has been presented by [112], relying on the location of the red clump giants in the infrared color-magnitude diagram and on the combination of reddening-distance relations with independent reddening estimates. The estimated distances have been checked against the distances measured with measured expansion parallax [47].

Gaia has changed the landscape of distances in astronomy [55], [56], [12]. The Gaia data release DR1 produced parallax estimations for three old novae, out of 16 cataclysmic variables [131]. The Gaia data release DR2 includes parallaxes for more than one thousand cataclysmic variables, among them several novae. The Gaia DR2 distance of 18 novae has been used by [146] to derive a new MMRD relation. The work by [142] has compared the Gaia parallaxes of 41 novae with previous distance estimates.

Novae have been considered as candidate standard candles in the estimation of extragalactic distances [181], [45]. The Very Large Telescope (VLT) has been used to search for novae as far as 
NGC 1316, an early type galaxy in the Fornax cluster [41] at about $20 \mathrm{Mpc}$ distance.

\section{Conclusions}

Novae are multifrequency laboratories for accretion processes and for the understanding of cataclsymic variables. New observations with high time cadence and in the high energy domain are providing new tools to investigate the outburst stage and the long term evolution.

\section{Acknowledgments}

The author thanks the organizers for the invitation to the conference. Thanks to Ivan Bruni for the assistance and to the Time Allocation Committee of the Loiano telescope for the observing time.

\section{References}

[1] A. A. Abdo et al., Sci 329 (2010) 817.

[2] M. Ackermann et al., Sci 345 (2014) 554.

[3] M. Actis et al., ExA 32 (2001) 143.

[4] M. L. Ahnen et al., A\&A 582 (2015) 582.

[5] C. W. Allen, MNRAS 114 (1954) 387.

[6] H. C. Arp et al., AJ 61 (1956) 15.

[7] D. P. K. Banerjee and N. M. Ashok, BASI 40 (2012) 243.

[8] M. F. Bode et al., ApJ 652 (2006) 629.

[9] M. F. Bode at al., ApJ 665 (2007) L63.

[10] M. F. Bode and A. Evans, Classical Novae, Cambridge University Press (2012).

[11] D. Boyd et al., ATel 11116 (2017).

[12] A. G. A. Brown et al.. A\&A 616 (2018) A1.

[13] W. Buscombe and G. de Vaucouleurs, Obs 75 (1955) 170.

[14] S. Buson et al., ATel 13114 (2020).

[15] M. Capaccioli et al., AJ 97 (1989) 1622.

[16] M. Capaccioli et al., ApJ 360 (1990) 63.

[17] A. Cassatella and R. Viotti, Physics of Classical Novae, Springer-Verlag, Lecture Notes in Physics 369 (1990).

[18] O. Chesneau et al., $A \& A 534$ (2011) L11.

[19] O. Chesneau et al., A\&A 545 (2012) A63.

[20] O. Chesneau and D. P. K. Banerjee, BASI 40 (2012) 267.

[21] C. C. Cheung et al., ApJ 826 (2016) 142. 
[22] C. C. Cheung et al., ATel 9594 (2016).

[23] L. Chomiuk et al., ApJ 761 (2012) 173.

[24] L. Chomiuk et al., Nat 514 (2014) 339.

[25] R. Ciardullo et al., ApJ 318 (1987) 520.

[26] R. Ciardullo et al., ApJ 356 (1990) 472.

[27] D. D. Clayton and F. Hoyle, ApJ 187 (1974) 101.

[28] J. G. Cohen, ApJ 292 (1985) 90.

[29] J. G. Cohen, in: The extragalactic distance scale, ASPC 4 (1988) 114.

[30] A. C. Collazzi et al., AJ 138 (2009) 1846.

[31] C. Curtin et al., ApJ 811 (2015) 34.

[32] I. Czekala et al., ApJ 765 (2013) 57.

[33] M. J. Darnley et al., MNRAS 369 (2006) 257.

[34] M. J. Darnley et al., ApJ 833 (2016) 149.

[35] G. de Vaucouleurs, ApJ 223 (1978) 351.

[36] M. Della Valle, in: The extragalactic distance scale, ASPC 4 (1988) 73.

[37] M. Della Valle et el., A\&A 266 (1992) 232.

[38] M. Della Valle et al., A\&A 287 (1994) 403.

[39] M. Della Valle, $A \& A 252$ (1991) L9.

[40] M. Della Valle, in Classical Nova Explosions, AIPC 637 (2002) 443.

[41] M. Della Valle and R. Gilmozzi, Sci 296 (2002) 1275.

[42] M. Della Valle and L. Izzo, arXiv:2004.06540v2.

[43] M. Della Valle and M. Livio, A\&A 286 (1994) 786.

[44] M. Della Valle and M. Livio, ApJ 423 L31.

[45] M. Della Valle and M. Livio, ApJ 452 (1995) 704.

[46] M. Della Valle and M. Livio, ApJ 506 (1998) 818.

[47] R. A. Downes and H. W. Duerbeck, AJ 120 (2000), 2007.

[48] R. A. Downes et al., JAD 11 (2005) 2

[49] H. W. Duerbeck, SSR 45 (1987) 1.

[50] H. W. Duerbeck, MNRAS 258 (1992) 629.

[51] H. W. Duerbeck, AN 330 (2009) 568.

[52] A. Ederoclite, in: Stella Novae: Past and Future Decades, ASPC 490 (2014) 163.

[53] A. Evans and R. D. Gehrz, BASI 40 (2012) 213.

[54] S. P. S. Eyres et al., ASP Conf. Ser. 93 (1996) 194.

[55] Gaia Collaboration et al., A\&A 595 (2016) A1. 
[56] Gaia Collaboration et al., $A \& A 616$ (2018) A1.

[57] T. R. Geballe et al., ApJL 886 (2019) L14.

[58] S. L. Geisel et al., ApJ 161 (1970) L101.

[59] F. Giovannelli and L. Sabau-Graziati, in Proceedings of The Golden Age of Cataclysmic Variables and Related Objects - III, POS (Golden2015) 001 (2016).

[60] J. A. Graham, in Changing Trends in Variable Star Research, IAU Coll. 46 (1979) 96.

[61] M. A. Guerrero et al., ApJ 857 (2018) 80.

[62] I. Hachisu and M. Kato, ApJSS 167 (2006) 59.

[63] I. Hachisu and M. Kato, ApJ 709 (2010) 680.

[64] K. Hatano et al., MNRAS 290 (1997) 113.

[65] M. Henze et al., A\&A 563 (2014) A2.

[66] N. Henze et al., $A \& A \mathbf{5 8 2}$ (2015) 8.

[67] M. Hernanz, in Stella Novae: Past and Future Decades, ASPC 490 (2014) 319.

[68] R. M. Hjellming et al., AJ 84 (1979) 1619.

[69] R. Hounsell et al., ApJ 734 (2010) 480.

[70] R. Hounsell et al., ApJ 820 (2016) 104.

[71] E. P. Hubble, ApJ 69 (1929) 103.

[72] R. Hudec, in Proceedings of The Golden Age of Cataclysmic Variables and Related Objects - III, PoS (Golden2015) 041 (2016).

[73] K. Imamura and K. Tanabe, PASJ 64 (2012) L9.

[74] K. Itagaki et al., ATel 9848 (2016).

[75] L. Izzo et al., ApJL 808 (2015) L14.

[76] P. Jean et al., ATel 11546 (2018).

[77] M. M. Kasliwal et al., ApJ 735 (2011) 94.

[78] M. Kato and I. Hachisu, ApJ 437 (1994) 803.

[79] I. M. Kopylov, IzvKrymAstr Obs 13 (1955) 23.

[80] J. Krautter et al., ApJ 456 (1996) 788.

[81] K.-L. Li et al., ATel 9736 (2016).

[82] K.-L. Li et al., NatAs 1 (2017) 697.

[83] K.-L. Li et al., ATel 10977 (2017).

[84] K.-L. Li et al., ATel 11590 (2018).

[85] K.-L. Li et al., ATel 13116 (2020).

[86] W. Liller and B. Mayer, PASP 99 (1987) 600.

[87] M. Livio, ApJ 393 (1992) 516. 
[88] K. Lundmark, MeLuS 74 (1935) 1.

[89] P. Martin et al., A\&A 612 (2018) A38.

[90] C. G. Mason et al., ApJ 494 (1998) 783.

[91] F. Matteucci et al., $A \& A 405$ (2003) 23.

[92] B. D. Metzger et al., MNRAS 450 (2015) 2739.

[93] B. D. Metzger et al., MNRAS 457 (2016) 1786.

[94] B. Miszalski et al., MNRAS 456 (2016) 633.

[95] P. Molaro et al, MNRAS 463 (2016) L117.

[96] P. J. Morris et al., MNRAS 495 (2017) 1218.

[97] P. Mroz et al., ApJS 219 (2015) 26.

[98] P. Mroz et al., ApJS 232 (2016) 9.

[99] P. Mroz et al., Nat 537 (2016) 649.

[100] https://asd.gsfc.nasa.gov/Koji.Mukai/novae/novae.html

[101] U. Munari et al., MNRAS 469 (2017) 4341.

[102] J. U. Ness et al., ApJ 663 (2007) 505.

[103] J. U. Ness, BASI 40 (2012) 353.

[104] T. J. O’Brien et al., Nat 442 (2006) 279.

[105] H. Ögelman et al., A\&A 177 (1987) 110.

[106] M. Orio et al., ApJ 466 (1995) 410.

[107] M. Orio, BASI 40 (2012) 333.

[108] M. Orio, in Proceedings of The Golden Age of Cataclysmic Variables and Related Objects - III, PoS (Golden2015) 064 (2016).

[109] M. Orio et al., ApJ 895 (2020) 80.

[110] J. P. Osborne eta al., ApJ 727 (2011) 124.

[111] J. P. Osborne, JHEA 7 (2015) 117.

[112] A. Özdönmez et al., MNRAS 461 (2016) 1177.

[113] K. Page et al., MNRAS 454 (2015) 3108.

[114] A. Pagnotta, in 20th European White Dwarf Workshop, ASPC 509 (2017) 535.

[115] J. Patterson, SASS 33 (2014) 17.

[116] C. H. Payne-Gaposchkin, The Galactic Novae, North-Holland Pub. Co. (1957).

[117] R. Poggiani, AN 327 (2006) 895.

[118] R. Poggiani, NewA 13 (2008) 557.

[119] R. Poggiani, Ap\&SS 315 (2008) 79.

[120] R. Poggiani, NewA 14 (2009) 4. 
[121] R. Poggiani, AN 330 (2009) 77.

[122] R. Poggiani, Ap\&SS 323 (2009) 319.

[123] R. Poggiani, NewA 15 (2010) 657.

[124] R. Poggiani, NewA 15 (2010) 170.

[125] R. Poggiani, Ap\&SS 333 (2011) 115.

[126] R. Poggiani, MmSAI 83 (2012) 753.

[127] R. Poggiani, NewA 37 (2015) 9.

[128] R. Poggiani, AcPPP 2 (2015) 234.

[129] R. Poggiani,in: Proceedings of The Golden Age of Cataclysmic Variables and Related Objects IV (GOLDEN 2017), POS (GOLDEN 2017$) 049$ (2018).

[130] R. Poggiani,in: Proceedings of XII Multifrequency Behaviour of High Energy Cosmic Sources Workshop (MULTIF2017) - Astrophysics of High Energy Cosmic Sources, POS (MULTIF2017) 053 (2018).

[131] G. Ramsay et al., $A \& A 604$ (2017) A107.

[132] H. Ritter and U. Kolb, A\&A 404 (2003) 301.

[133] http://wwwmpa.mpa-garching.mpg.de/RKcat/

[134] E. L. Robinson, AJ 80 (1975) 515.

[135] L. Rosino, AnAp 27 (1964) 498.

[136] L. Rosino, A\&AS 9 (1973) 347.

[137] L. Rosino, AJ 97 (1989) 83.

[138] N. Roy et al., BASI 49 (2012) 293.

[139] D. I. Sahman et al., MNRAS 477 (2018) 4483.

[140] B. E. Schaefer, ApJS 187 (2010) 275.

[141] G. H. Schaefer et al., Nat 515 (2014) 234.

[142] B. E. Schaefer, MNRAS 481 (2019) 3033.

[143] T. Schmidt, Z. Astrophys. 41 (1957) 182.

[144] G. J. Schwarz et al., MNRAS 320 (2001) 103.

[145] G. Schwarz et al., ApJS 197 (2011) 31.

[146] P. Selvelli and R. Gilmozzi, A\&A 622 (2019) 186.

[147] A. W. Shafter, ApJ 487 (1997) 226.

[148] A. W. Shafter et al., ApJ 530 (2000) 193.

[149] A. W. Shafter, in: Classical Nova Explosions: International Conference on Classical Nova Explosions, AIPC 637 (2002) 462.

[150] A. W. Shafter et al., ApJ 690 (2009) 1148

[151] A. W. Shafter et al., ApJ 734 (2011) 12. 
[152] A. W. Shafter et al., ApJ 752 (2012) 156.

[153] A. W. Shafter, AJ 145 (2013) 117.

[154] A. W. Shafter, in: Stella Novae: Past and Future Decades, ASPC 490 (2014) 77.

[155] A. W. Shafter et al., ApJS 216 (2015) 34.

[156] A. W. Shafter, ApJ 834 (2017) 196.

[157] A. W. Shafter, Extragalactic Novae, IOP Publishing (2019).

[158] A. W. Shafter and P. K. Irby, ApJ 563 (2001) 749.

[159] M. M. Shara et al., ApJ 311 (1986) 163.

[160] M. M. Shara et al., Nat 446 (2007) 159.

[161] M.M. Shara et al.,ApJ 758 (2012) 121.

[162] M. M. Shara et al., ApJS 227 (2016) 1.

[163] M. M. Shara et al., Nat 548 (2017) 558.

[164] M. M. Shara et al., ApJ 839 (2017) 109.

[165] M. M. Shara et al., MNRAS 465 (2017) 739.

[166] M. M. Shara et al., MNRAS 474 (2018) 1746.

[167] A. S. Sharov, SvA 16 (1972) 41.

[168] A. S. Sharov, AstL 19 (1993) 147.

[169] J. Sitarek and W. Bednarek, PRD 86 (2012) 063011.

[170] A. J. Slavin et al., MNRAS 276 (1995) 353.

[171] J. J. Sokoloski et al., Nat 442 (2006) 276.

[172] S. Starrfield et al., PASP 128 (2016) 051001.

[173] S. Starrfield, these proceedings.

[174] R. J. Strope et al., AJ 140 (2010) 34.

[175] F. Surina et al., in The 11th Asian-Pacific Regional IAU Meeting 2011 NARIT Conf. Ser. 1 (2013) 161.

[176] A. Tajitsu et al., Nat 518 (2015) 381.

[177] J. Tanaka et al., PASJ 63 (2011) 911.

[178] C. Tappert et al., MNRAS 423 (2012) 2476.

[179] C. Tappert et al., in: Proceedings of The Golden Age of Cataclysmic Variables and Related Objects III, PoS (Golden2015) 062 (2016).

[180] A. B. Tomaney and A. W. Shafter, ApJS 81 (1992) 683.

[181] S. van den Bergh and C. J. Pritchet, PASP 98 (1986) 110.

[182] S. van den Bergh and P. F. Younger, A\&AS 70 (1987) 125.

[183] S. van den Bergh, PASP 103 (1991) 609. 
[184] I. Vurm and B. D. Metzger, ApJ 852 (2018) 62.

[185] R. A. Wade eta al., PASP 112 (2000) 614.

[186] R. Wagner et al., AAS Meeting \#231, id. 358.10.

[187] F. M. Walter et al., PASP 124 (2012) 1057.

[188] F. M. Walter, in Stella Novae: Past and Future Decades, ASPC 490 (2014) 191.

[189] R. E. Williams et al., ApJ 376 (1991) 721.

[190] R. E. Williams, $A J 104$ (1992) 725.

[191] R. E. Williams et al., ApJSS 90 (1994) 297.

[192] R. E. Williams, JAD 9 (2003).

[193] R. E. Williams and A. W. Shafter, ApJ 612 (2004) 867.

[194] R. Williams, AJ 144 (2012) 98.

[195] O. Yaron et al., ApJ 623 (2005) 398.

[196] L. Yungelson et al., ApJ 481 (1997) 127. 